\title{
Characterisation of bronchus-associated lymphoid tissue and antigen-presenting cells in central airway mucosa of children
}

\author{
Ingvild Heier, ${ }^{1,2}$ Kristiina Malmström, ${ }^{3}$ Antti Sajantila, ${ }^{4}$ Jouko Lohi, ${ }^{5}$ Mika Mäkelä, ${ }^{3}$ \\ Frode L Jahnsen ${ }^{1,6}$
}

${ }^{1}$ LIIPAT, Institute of Pathology and Centre for Immune Regulation, University of Oslo and Oslo University Hospital, Oslo, Norway

${ }^{2}$ Department of Pediatrics, Oslo University Hospital, University of Oslo, Oslo, Norway

${ }^{3}$ Department of Allergy, Helsinki University Central Hospital, Helsinki, Finland

${ }^{4}$ Department of Forensic Medicine, Hjelt Institute University of Helsinki, Helsinki, Finland

${ }^{5}$ Department of Pathology, Helsinki University Central Hospital, Helsinki, Finland ${ }^{6}$ Department of Pathology, Oslo University Hospital, Oslo, Norway

\section{Correspondence to}

Frode L Jahnsen, Department of Pathology, Oslo University Hospital, Rikshosptalet, Postboks 4950 Nydalen, 0424 Oslo, Norway; frode.lars. jahnsen@rr-research.no

Received 23 August 2010 Accepted 10 November 2010 Published Online First 16 December 2010

\begin{abstract}
Background Childhood represents an immunological window of vulnerability in which individuals are at increased risk for both serious infections and development of allergic diseases, particularly affecting the airways. However, little is known about how the airway mucosal immune system is organised and functions during early age. Here, the organisation of immune cells in bronchial mucosa of children was characterised.
\end{abstract}

Methods Immunophenotyping was performed on mucosal samples obtained postmortem from nine children aged 2-15 years without any history of atopic manifestations or any signs of respiratory disease, who died from non-inflammatory causes.

Results In all nine cases, isolated lymphoid follicles (ILFs), interpreted as bronchus-associated lymphoid tissue (BALT), were found, constituting an average frequency of $60 \mathrm{ILFs} / \mathrm{cm}^{2}$ of airway mucosal surface. Outside these ILFs, dense networks of $\mathrm{CD}_{11 \mathrm{c}^{+}}$myeloid dendritic cells (DCs), $\mathrm{CD}^{+} 8^{+}$macrophages and $\mathrm{CD}^{+}{ }^{+}$CD45RA ${ }^{-}$memory $T$ cells were found.

Plasmacytoid DCs occurred in low numbers. Importantly, intraepithelial antigen-presenting cells were found to extend cellular projections into the airway lumen. Conclusion The density and location of antigenpresenting cells and T cells in this age group are similar to those observed in adults. However, in contrast to adults, BALT appears to be a normal feature of the airway mucosa throughout childhood, suggesting that these structures contribute to regional immunity and homeostasis. This indicates that the local immune system in the airways of children has unique features which should be taken into account, not only when studying airway immunology and immunopathology, but also in the development of mucosal vaccines.

\section{INTRODUCTION}

The respiratory tract has a large mucosal surface that is continuously exposed to a range of antigens, both pathogenic microbes and innocuous material, such as dust and pollen. The local immune system therefore faces a considerable challenge in order to discriminate between pathogenic antigens that merit rapid elimination and harmless ubiquitous antigens which need to be 'tolerated' to avoid chronic inflammatory damage. ${ }^{1}$ Epidemiological studies have shown that in preschool years individuals are more susceptible to both respiratory viral infections and aeroallergen sensitisation than later in life. ${ }^{2}{ }^{3}$ These findings suggest that there is a relative dysfunction of the airway immune system during the first years of life. At birth, the adaptive immune response appears to be attenuated and skewed towards production of $\mathrm{T}$ helper 2 (Th2) cytokines with a relative inability to produce Th1 immunity. ${ }^{3}$ This postnatal Th1/Th2 imbalance may increase the risk of developing Th2-dependent allergic immunopathology and at the same time increase the risk for viral infections due to insufficient Th1 responses. However, although there is a Th2 skewing in early life, the majority of infants do not become sensitised to inhaled antigens, showing that effective regulatory mechanisms exist. ${ }^{4}$ This suggests that there may be functional differences at the level of the local mucosal immune system in the respiratory tract. However, little is known about how the airway mucosal immune system is organised and functions during early age. ${ }^{1}$

Mouse models have shown that airway mucosal dendritic cells (AMDCs) are the main inducers of adaptive immunity in the respiratory tract. ${ }^{15}$ They are strategically situated at mucosal surfaces where they continuously sample antigen from the environment. Antigen-bearing AMDCs continuously migrate to the draining lymphoid nodes where they present antigenic material to naïve $\mathrm{T}$ cells and initiate productive immune responses. ${ }^{5} 6$ AMDCs consist of several subsets which are functionally distinct. Classical CD11c ${ }^{+}$myeloid DCs have been shown to initiate $T$ cell responses to both viruses ${ }^{7}$ and aeroallergens. ${ }^{8}$ The rare plasmacytoid (p)DCs, characterised by high expression of CD123, are also important in antiviral immunity through their ability to produce large amounts of interferon $\alpha$ (IFN $\alpha) .{ }^{9}$ However, with respect to allergens, this cell population has been associated with induction of tolerance. ${ }^{10-12}$ The distribution of DC subsets is therefore relevant to local immune functions. This notion is underscored by the recent finding that in children with a family history of atopy, lower numbers of circulating pDCs were associated with more frequent and more severe respiratory tract infections, wheezing and a diagnosis of asthma. ${ }^{13}$

An apparent difference between the airway immune system in infants compared with adults is the presence of bronchus-associated lymphoid tissue (BALT). ${ }^{14} 15$ BALT is part of the mucosaassociated lymphoid tissue and is defined as organised lymphoid aggregates within the bronchial mucosa in contact with the surface epithelium. ${ }^{15}$ The occurrence of BALT differs between species. It was first described in rabbits ${ }^{16}$ where it is regularly present, as opposed to mice in which 
BALT is only occasionally observed after birth. However, interestingly, mice deficient for the chemokine receptor CCR7 regularly developed organised BALT from day 5 onwards, independently of an immunological insult. ${ }^{17}$ This was shown to depend on impaired recruitment of regulatory $T$ (Treg) cells to lung-draining bronchial lymph nodes, suggesting that Treg cells were involved in the control of BALT formation. In mice, BALT is also induced by infection or inflammation; so-called inducible BALT (iBALT). Importantly, it was recently shown that adaptive immune responses that were generated in iBALT against influenza virus were not only protective but also less pathogenic than systemic immune responses. ${ }^{18}$ Little is known about the function of these lymphoid structures in humans. BALT is not regularly found in fetuses, as opposed to the constitutive Peyer's patches of the intestine, but is probably induced by antigen stimulation after birth. ${ }^{19}$ BALT in adults has primarily been associated with various diseases, ${ }^{15}$ but we and others have shown that it is found more regularly during childhood. ${ }^{15} 1920$ To understand how adaptive response mechanisms operate in the airways of children, both the function of AMDC subsets and the participation of BALT should be taken into account.

We have recently characterised the distribution of immune cells in biopsy material from bronchial mucosa of infants $<2$ years of age suffering from respiratory symptoms. ${ }^{20}$ We found that there was a tight network of antigen-presenting cells (APCs) at the mucosal surface and that isolated lymphoid follicles (ILFs), representing components of BALT, were present in half of the samples. However, for obvious ethical reasons we were not able to obtain biopsies from normal individuals. To characterise the organisation of the bronchial immune system at baseline we have performed a detailed characterisation of the distribution and functional phenotype of APCs as well as the occurrence of BALT in the bronchial mucosa of children who died from non-infectious causes, with no history of atopy or airway disease.

\section{MATERIALS AND METHODS Subjects}

Specimens from the lower part of the trachea including the bifurcation and proximal left and right bronchus were obtained from nine children ( 7 caucasian and 2 oriental) aged 1.9-15.4 years (median 8.5) who died from traumatic causes (3 traffic accidents, 4 other accidents and 2 submersions). The median time from death to autopsy was 4 days (range 1-9). Pulmonary contusion, oedema, intrapulmonary and intrabronchial haemorrhage were observed at the medico-legal autopsies in most of the samples related to the mechanism of death. While mild autolytic changes were noted in half of the specimens, only one specimen had undergone significant autolysis of the submucosal tissue. There were no inflammatory or respiratory disease-specific findings. The health charts from public and private healthcare producers were carefully studied. None of the individuals had a history of wheezing disorders, asthma and/or other atopy-related manifestations. The study was approved by the local ethics committee in Helsinki and the National Supervisory Authority for Welfare and Health in Finland.

\section{Immunohistochemistry}

Specimens were formalin fixed and paraffin embedded. Sections cut at $4 \mu \mathrm{m}$ were examined by immunohistochemistry. For optimal staining results, heat-induced epitope retrieval was performed by boiling the sections in a microwave oven for $20 \mathrm{~min}$ in citrate buffer ( $\mathrm{pH} 6.0)$.
Immunoenzyme staining on dewaxed tissue sections was performed in a Ventana NexEs IHC instrument (Tucson, Arizona, USA) with the standardised iView DAB or enhanced $\mathrm{V}$-Red (alkaline phosphatase) detection kits as recommended by the manufacturer. The primary monoclonal antibodies (mAbs) were directed against human HLA-DR (clone TAL.1B5, immunoglobulin G1 (IgG1), DakoCytomation, Glostrup, Denmark), CD68 (clone PG-M1, IgG3, DakoCytomation), CD11c (clone 5D11, IgG2a, Novocastra Lab, Newcastle Upon Tyne, UK), CD1a (clone MTB1, IgG1, Novocastra), CD123 (a mixture of clone 7G3, IgG2a, and clone 9F5, IgG1, BD Pharmingen, San Diego, California, USA), CD45RA (clone L48, BD Immunocytometry Syst., San Jose, California, USA) and CD20 (clone L26, IgG2a, DakoCytomation). A polyclonal rabbit antihuman CD3 (dilution 1/50; DakoCytomation) was additionally applied. To ensure that $\mathrm{CD} 23^{+}$cells were $\mathrm{pDCs}$, we performed additional experiments by means of multicolour immunofluorescence staining in which we examined the cellular co-expression of CD123 with HLA-DR and CD45RA. This method has been described in detail elsewhere. ${ }^{21}$

Stained tissue sections were examined by the same investigator $(\mathrm{IH})$ at $\times 400$ magnification. The cell density in the epithelium was recorded as the total number of positive cell profiles per basement membrane length unit $(1 \mathrm{~mm})$, whereas the cell number in the lamina propria was recorded as positive cell profiles per square millimetre. The total number of positive mucosal cells was recorded per basement membrane length unit by adding the intraepithelial counts and the lamina propria counts to a depth of the grid. On average, 50 visual fields were counted in each specimen. The mucosal areas containing lymphoid aggregates (see Results section) were omitted from the cell enumeration.

Stainings for the nuclear antigens FOXP3 and Ki67 with antibodies that normally work well on paraffin sections were unsuccessful, possibly due to postmortem degradation of nuclear proteins.

Sections were also stained with H\&E for histological evaluation and with period acid-Schiff to identify goblet cells.

\section{RESULTS \\ Histological evaluation}

H\&E staining showed horizontally cut sections through the whole bronchial wall including parabronchial lymph nodes. The morphology in most specimens was very good, with a well preserved surface epithelium in seven out of nine samples. In some sections the epithelium was partly detached from the underlying lamina propria. There was no accumulation of neutrophils or eosinophils or other signs of ongoing inflammation. In nearly all sections lymphoid aggregates were observed, mostly in close proximity to the surface epithelium, but also deeper in the lamina propria and submucosa.

\section{BALT was a prominent feature in all subjects}

Lymphoid aggregates in contact with the epithelium were recognised as putative ILFs representing components of BALT. These were therefore further characterised by immunostaining experiments applying a range of $\mathrm{mAbs}$ to identify subsets of immune cells. All ILFs contained high numbers of HLA-DR ${ }^{+}$ cells, $\mathrm{CD}^{+} \mathrm{T}$ cells and $\mathrm{CD} 20^{+} \mathrm{B}$ cells (figure $1 \mathrm{~A}-\mathrm{D}$ ). Most $\mathrm{T}$ cells were of the naïve phenotype, judged by the dense population of $\mathrm{CD}_{45 \mathrm{RA}^{+}}$cells (figure 1E,F). CD11c ${ }^{+} \mathrm{DCs}$, but not $\mathrm{CD}_{6}{ }^{+}$macrophages, accumulated in the ILFs (figure 2A and not shown). Moreover, small numbers $\mathrm{CD}_{123^{+}}$cells, 
presumably pDCs, were observed (figure 2B). By two-colour immunofluorescence staining the $\mathrm{CD}_{12} 3^{+}$cells co-expressed both HLA-DR and CD45RA, demonstrating that they indeed were pDCs (not shown). Some of the vessels within these lymphoid aggregates expressed MECA-79, demonstrating that the ILFs contained high endothelial venules (HEVs) (figure 2C). We also observed very few $\mathrm{CD}_{2}{ }^{+}$follicular DCs within these aggregates (not shown). Moreover, no goblet cells (period acidSchiff-positive cells) were detected in the epithelium covering ILFs. Together, these findings demonstrated that these lymphoid structures clearly could be defined as BALT.

The numbers of ILFs were counted in sections stained for CD3/CD20 and CD45RA, which clearly delineated these structures (figure $1 \mathrm{C}-\mathrm{F}$ ). Sections from all individuals contained ILFs with a median of four per section (range 1-11). The density of ILFs per millimetre basement membrane was determined by dividing the number of ILFs by the bronchial circumference (figure 3). With an estimated median ILF diameter of $200 \mu \mathrm{m}$, we calculated that the density of ILFs was $\sim 60 / \mathrm{cm}^{2}$ of airway mucosal surface. This finding showed that BALT is a prominent feature of the distal trachea/proximal bronchus during childhood.

\section{Immune cell populations outside organised lymphoid tissue}

Apart from ILFs, HLA-DR ${ }^{+}$cells were evenly distributed throughout the circumference, both within and beneath the surface epithelium, constituting a dense network of large cells with variable morphology. Within the epithelium the median number of HLA-DR ${ }^{+}$cells per mm basement membrane was 34 (range 14-56; figure 4A), whereas in the lamina propria a median number of 342 HLA-DR ${ }^{+}$cells per $\mathrm{mm}^{2}$ (range 172-424; figure 4B) was found. Interestingly, intraepithelial HLA-DR ${ }^{+}$cells frequently extended cell projections through the epithelium into the luminal side (figure 5A). There was no correlation between the number of HLA-DR ${ }^{+}$cells and age (figure 4C).

To characterise the APC populations further we determined the number of $\mathrm{CD}^{+} 8^{+}$macrophages and $\mathrm{CD}_{11 c^{+}}$putative myeloid DCs. The number of $\mathrm{CD}^{+} 8^{+}$and $\mathrm{CD}_{11 c^{+}}$cells was quite similar both in the epithelium and in the lamina propria, and the sum of these cell populations was close to $100 \%$ of all $\mathrm{HLA}_{-} \mathrm{DR}^{+}$cells in the lamina propria and $60-70 \%$ of all HLA$\mathrm{DR}^{+}$cells in the epithelial compartment (figure 4A,B). Additional co-staining experiments showed that there was only $\sim 15 \%$ overlap between the two markers (figure $5 \mathrm{~B}$ ), indicating that the mucosal APC populations were mainly made up of $\mathrm{CD}_{68}{ }^{+}$macrophages and $\mathrm{CD} 11 \mathrm{c}^{+} \mathrm{DCs}$. We and others have found that some AMDCs express CD1a, a marker used to identify epidermal Langerhans cells. ${ }^{20}$ In this material only low densities of $\mathrm{CD}^{+}{ }^{+}$cells were found both in the epithelium (median 0.07, range 0-1; figure 4A) and in the lamina propria (median 0.0, range 0.0-6; figure $4 \mathrm{~B}$ ). pDCs were also found
Figure 1 The bronchial mucosa of children contains isolated lymphoid follicles (ILFs). Immunoenzyme staining for human leucocyte antigen (HLA)-DR ( $A$ and B), CD3 (brown) and CD20 (red) ( $B$ and $C$ ) and CD45RA (E and F) of formalin-fixed and paraffin-embedded sections from the bronchial wall. Note distinct subepithelial cell aggregates (ILFs) for all markers applied (some arrowed). Magnification: $A, D, \times 100$; $B, \times 200 ; C, E, \times 40 ; F, \times 400$.
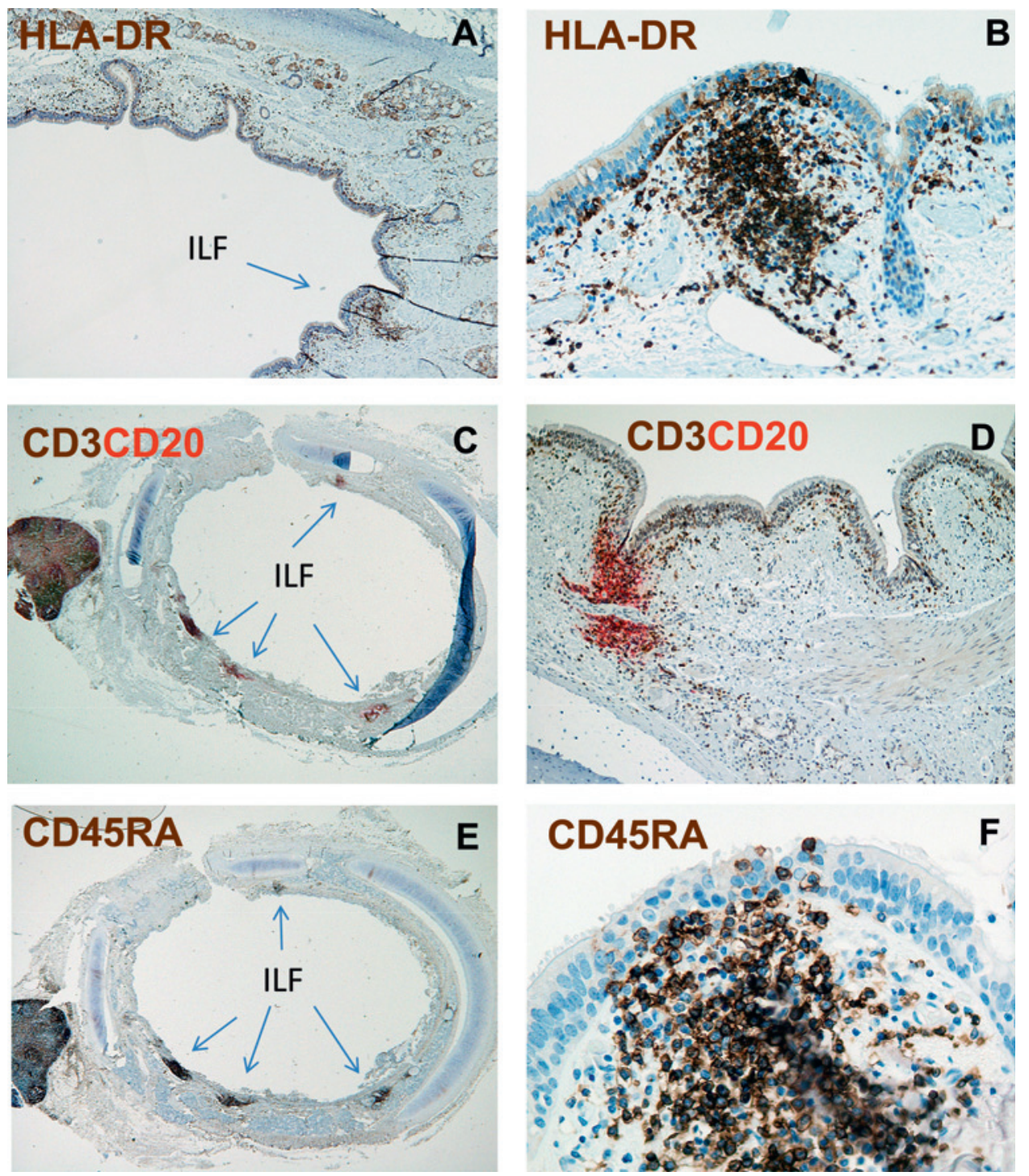

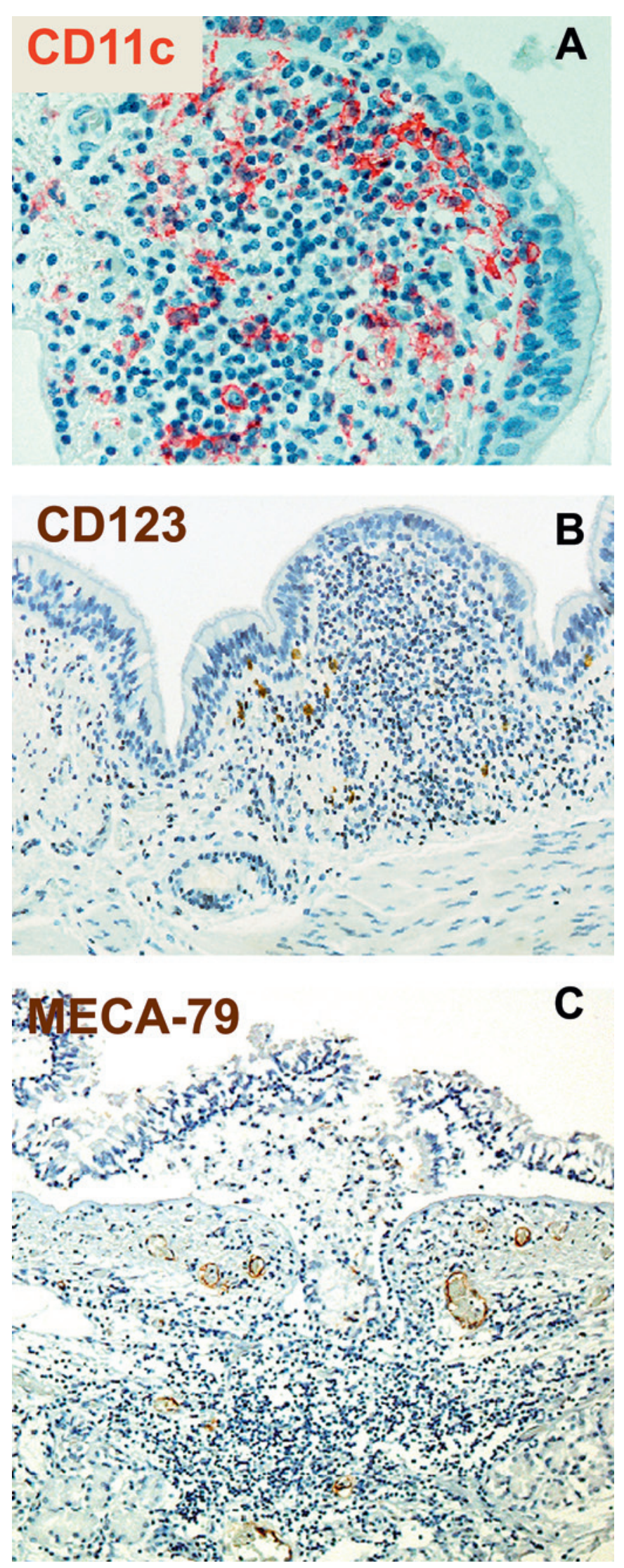

Figure 2 Isolated lymphoid follicles contain myeloid and plasmacytoid dendritic cells (DCs), and high endothelial venules. Immunoenzyme staining for CD11c (A), CD123 (B) and MECA-79 (C) of formalin-fixed and paraffin-embedded sections from the bronchial wall. Note the dendritic morphology of $\mathrm{CD}_{11 \mathrm{c}^{+}}$cells (A). Magnification: $A, \times 400 ; B, C, \times 200$.

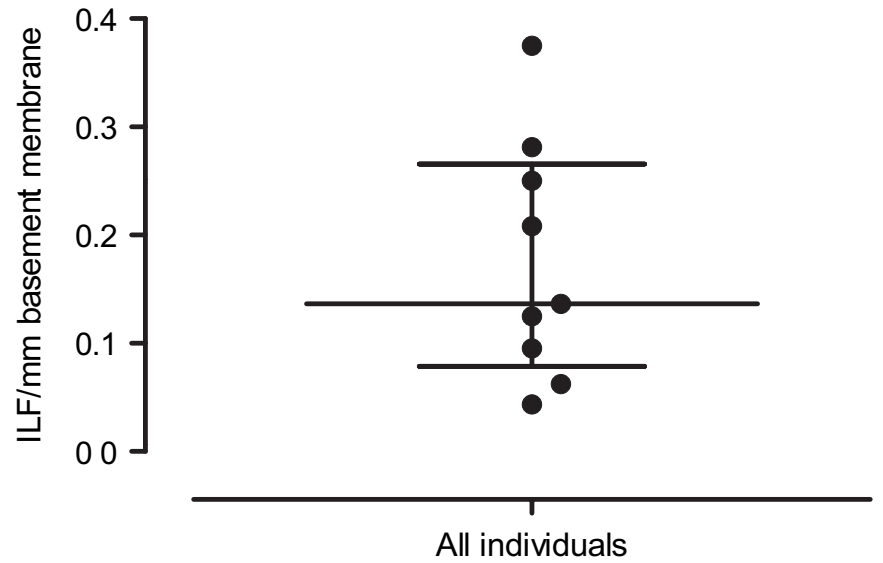

Figure 3 Bronchus-associated lymphoid tissue (BALT) was a prominent feature in the bronchial mucosa of children. Total numbers of isolated lymphoid follicles (ILFs) were counted in sections stained for CD45RA and CD3/CD20, and given as numbers of ILFs per millimetre basement membrane. Data are reported as median and IQR.

scattered in the lamina propria (median 7, range 2-24) and within the epithelium in some specimens (median 0.25, range $0-2$; figure $4 \mathrm{~A}, \mathrm{~B})$.

There was no correlation between the time of death to autopsy and the cell counts for any of the cell types (not shown).

\section{T and B cells outside organised lymphoid tissue}

$\mathrm{CD}^{+} \mathrm{T}$ cells were observed evenly distributed throughout the surface epithelium and lamina propria (figure 5C). However, the $\mathrm{CD}_{45 \mathrm{RA}^{+}}$phenotype was restricted to ILFs (figure 1E), demonstrating that mucosal $\mathrm{T}$ cells were CD45RA ${ }^{-}$memory/ effector cells. Only very few $\mathrm{CD} 20^{+} \mathrm{B}$ cells were found outside ILFs (figure 5C).

\section{DISCUSSION}

Here we show that DCs, macrophages and $\mathrm{T}$ cells constitute a dense network of immune cells both beneath and within the surface epithelium in the normal bronchial mucosa of children between 1 and 15 years of age, which is similar to that found in healthy adults. However, in contrast to adults, organised lymphoid tissue identified as BALT was found in all studied children and therefore appeared to be a normal constituent of the local immune system in this age group. This finding suggests that BALT may play an important role as an inductive site for mucosal immune responses during childhood.

We have previously determined the density of APC subsets in bronchial biopsies from 45 infants $<2$ years of age with respiratory symptoms. ${ }^{20}$ Both the anatomical distribution and density of $\mathrm{HLA}_{-} \mathrm{DR}^{+}$cells and $\mathrm{CD}^{+} 8^{+}$cells were similar to what were reported here. Since there were no signs of inflammation, assessed by infiltration of granulocytes, in any of these studies, the similar results suggest that they represent the homeostatic distribution of APCs in children. Here we also showed that the vast majority of $\mathrm{CD} 11 \mathrm{c}^{+}$cells were negative for CD68 and that only a few pDCs were present in the mucosa. This finding strengthens the notion that $\mathrm{CD} 11 \mathrm{c}^{+}$cells constitute the majority of the AMDC population. Individuals in the present study were older (2-15 years) than in our previous biopsy study $(0-2$ years). In this postmortem material there was no correlation between the total number of HLA-DR ${ }^{+}$cells and age, like we found in infants from $0-2$ years, suggesting that the number of AMDCs and macrophages reach steady-state levels at 
A

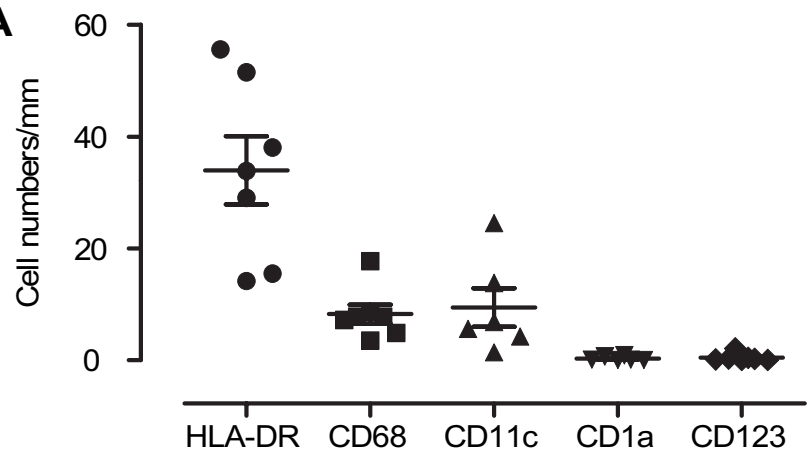

B

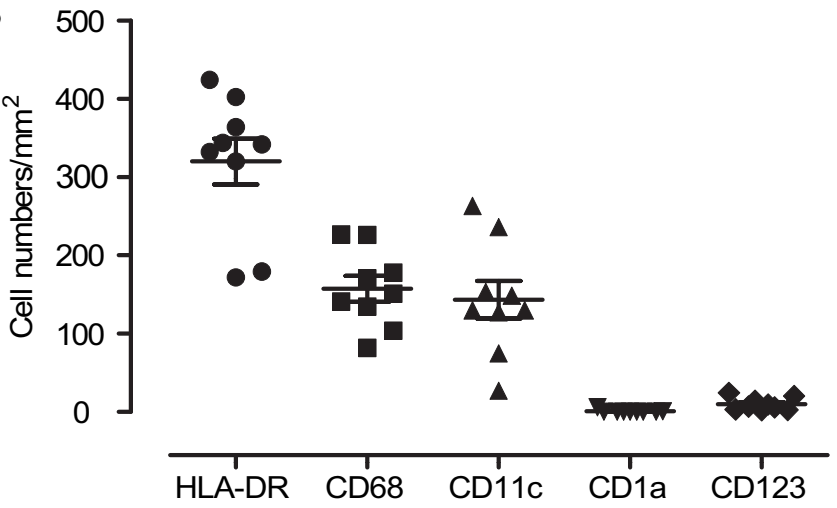

C

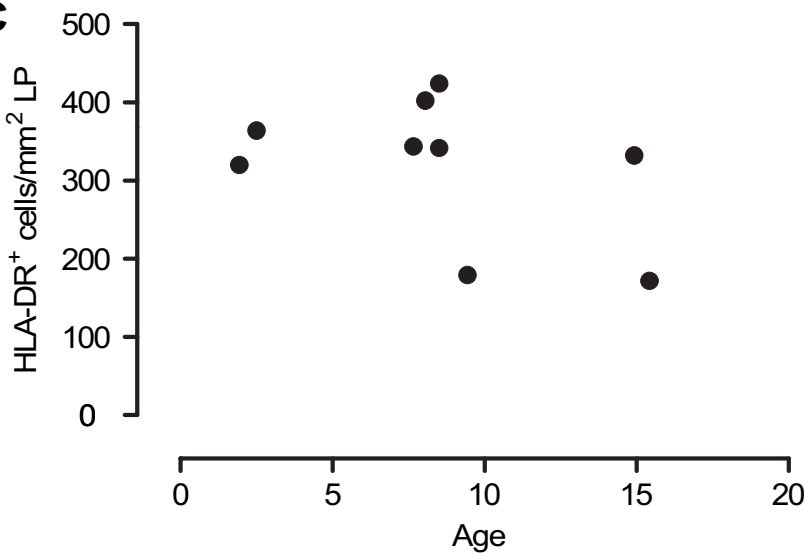

Figure 4 Density of antigen-presenting cell (APC) subsets in epithelium and in lamina propria. The density of human leucocyte antigen (HLA)-DR ${ }^{+}$, $\mathrm{CD}^{+} 8^{+}, \mathrm{CD} 11 \mathrm{c}^{+}, \mathrm{CD}^{\mathrm{a}} \mathrm{a}^{+}$and $\mathrm{CD} 123^{+}$cells in the epithelium $(\mathrm{A})$ and in the lamina propria $(B)$ were calculated per millimetre basement membrane and per square millimetre, respectively. There was no correlation between age and the number of $\mathrm{HLA}_{-} \mathrm{DR}^{+}$cells in the lamina propria (C).

$\sim 2$ years of age. This is in agreement with a recent study by Tschernig et al ${ }^{22}$ examining the density of tracheal APCs in a postmortem material. They found higher densities of tracheal APCs in those over 1 year of age compared with infants in their first year of life, and the density of HLA-DR ${ }^{+}$cells $(300-400 /$ $\mathrm{mm}^{2}$ ) in those $>1$ year (mostly adults) was very similar to what we found here.

DCs in the gut have been shown to sample bacterial antigen from the gut lumen in response to inflammatory stimuli. ${ }^{23}$ We observed here that 'snorkelling DCs' were regularly present, even in the absence of overt inflammation, suggesting that this is a means by which DCs, under homeostatic conditions, sample innocuous material for the induction of tolerance. ${ }^{1}$

We found that mucosal samples from all individuals contained dense aggregates of lymphoid tissue in close proximity to the
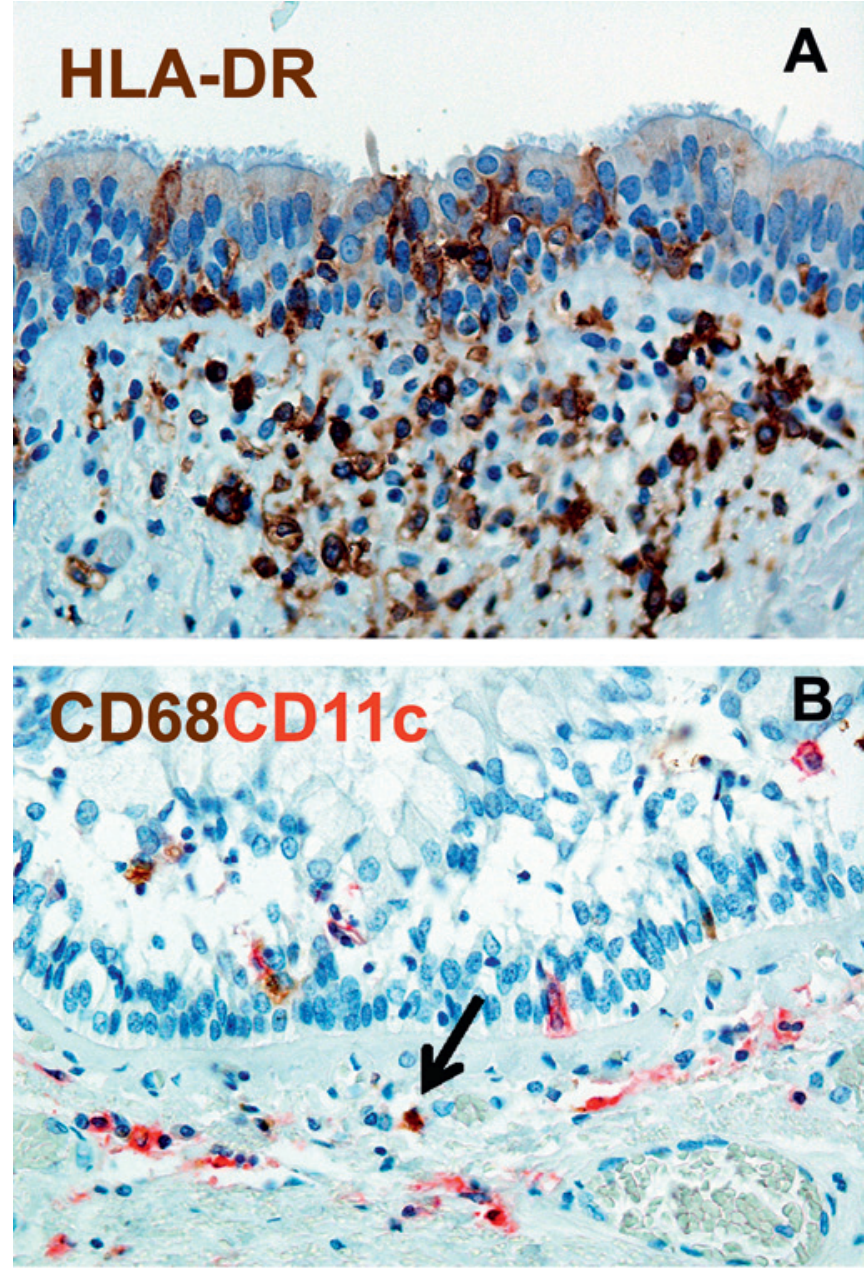

\section{CD3 CD20}

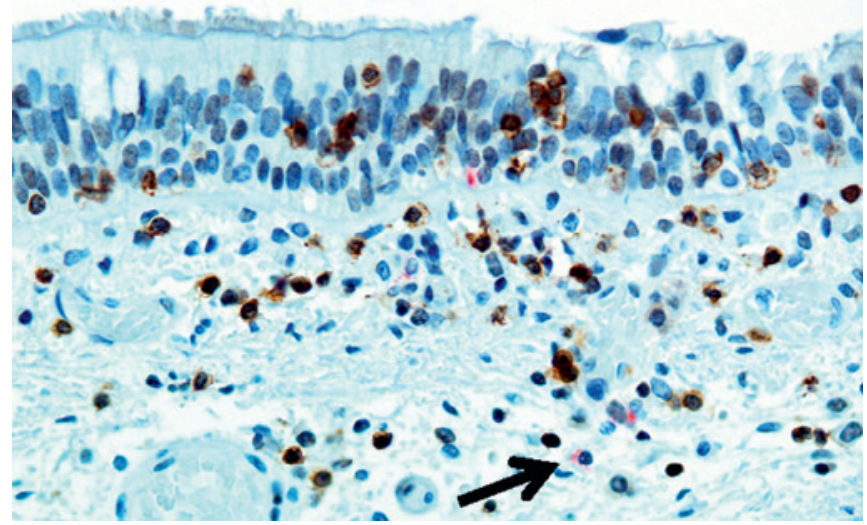

Figure 5 High densities of antigen-presenting cell (APC) subsets and T cells are found throughout the bronchial mucosa. Immunoenzyme staining for human leucocyte antigen (HLA)-DR (A), CD11c (red) and CD68 (brown) (B), and T (brown) and B (red) cells (C) of formalin-fixed and paraffin-embedded sections from the bronchial wall. Note HLA-DR ${ }^{+}$ cells with extensions reaching the luminal surface (A). Only very few cells co-express CD11c and CD68 (one arrowed) (B). Only very few B cells were identified in the bronchial mucosa (one arrowed) (C). Magnification: $\mathrm{A}-\mathrm{C}, \times 400$.

surface epithelium. These aggregates were heavily infiltrated by naïve T cells and HLA-DR ${ }^{+}$APCs. In addition, they consisted of $\mathrm{B}$ cells, CD11 $\mathrm{c}^{+}$myeloid DCs, pDCs, HEVs and follicular DCs, demonstrating that these ILFs indeed represented BALT. We also 
identified ILFs in lamina propria and submucosa that were not in contact with the surface epithelium. Such structures are not able to sample antigens directly from the airway lumen and were therefore not recognised as part of BALT. ${ }^{24}$ We estimated the density of ILFs as BALT to $\sim 60 / \mathrm{cm}^{2}$, demonstrating that these structures are an integral part of the mucosal immune system. In our biopsy study ${ }^{20}$ we identified BALT in $\sim 50 \%$ of the samples. Because biopsy specimens from the bronchial mucosa of infants were very small we would not expect to find BALT in more than half of the cases with a density of $60 / \mathrm{cm}^{2}$. Therefore, it seems that BALT develops within months after birth and is an essential component of the mucosal immune system in the bronchi during childhood. Tschernig et al found BALT in children but only in a fraction (44\%) of those who died from trauma. ${ }^{25}$ The difference between the latter report and ours could at least in part be due to the criteria used to define these structures. We identified BALT as aggregates of naïve $T$ and $B$ cells that were in contact with the overlying surface epithelium and showed that these structures also contained DCs and HEVs. Tschernig et al based their identification of BALT on routinely stained sections. In our experience, identification of BALT by immunostaining is a much more sensitive technique.

Because BALT is not regularly found in mice, most studies examining the airway immune system have emphasised the importance of AMDCs migrating to the draining lymph nodes to prime the adaptive immune system. We show here that in contrast to findings in mice, BALT is a prominent feature in children without any signs of airway inflammation. This may suggest that the immune responses to aeroantigens in children, both tolerance and immune activation, may be initiated to a large extent locally without the participation of the regional lymph nodes. Interestingly, experimental mice with iBALT that are lacking peripheral lymphoid organs cleared influenza viral infection and survived higher viral loads than normal mice, ${ }^{18} 26$ indicating an important protective role for iBALT. BALT should therefore be taken into account when studying the airway immune system and its importance for health and disease in children.

pDCs have been shown to be important inducers of tolerance to airway allergens in mouse models, ${ }^{12}$ and levels of pDCs during infancy were inversely correlated with symptoms of lower respiratory tract infections, parent-reported wheezing and the cumulative rate of physician-diagnosed asthma up to age 5 years. ${ }^{13}$ Here we confirm our previous findings that in airway mucosa of children, pDCs are primarily located within BALT, but in addition we show that they are also found scattered in the lamina propria and even within the epithelium, suggesting that they may play a role in mucosal homeostasis.

Studies have shown that the immune system in young children is immature, which may explain the high risk of viral infections and allergic sensitisation. ${ }^{3}$ To understand the function of the immune system, all structural features must be taken into account. This paper contributes to the understanding of the mucosal immune system in early life. APCs are strategically positioned within the epithelium and actively penetrate the epithelial barrier with cellular extensions, making these cells very suitable for sensing the luminal content of antigens. Moreover, BALT, similar to nasopharyngeal lymphoid tissue, should be recognised as an important inductive site in the respiratory tract. These aspects should be taken into account not only to understand airway immunology and immunopathology, but also in the development of mucosal vaccines. In line with this, a two-step vaccination protocol by inducing BALT in adults has recently been proposed. ${ }^{24}$
Acknowledgements We wish to thank laboratory technician Ge Qiu for processing the samples, and Hogne Røed Nilsen for excellent technical assistance with the immunohistochemical stainings.

Funding The study was supported by the Pediatric Department, Oslo University Hospital, Helsinki University Central Hospital Research Funds, Sigrid Juselius Foundation and the Finnish Allergy Reseach Foundation.

\section{Competing interests None.}

Ethics approval This study was conducted with the approval of the local ethics committee in Helsinki and the National Supervisory Authority for Welfare and Health in Finland.

Provenance and peer review Not commissioned; externally peer reviewed.

\section{REFERENCES}

1. Holt PG, Strickland DH, Wikstrom ME, et al. Regulation of immunological homeostasis in the respiratory tract. Nat Rev Immunol 2008;8:142-52.

2. Holgate ST. The epidemic of asthma and allergy. J $R$ Soc Med 2004;97:103-10.

3. Holt PG, Upham JW, Sly PD. Contemporaneous maturation of immunologic and respiratory functions during early childhood: implications for development of asthma prevention strategies. J Allergy Clin Immunol 2005:116:16-24.

4. Umetsu DT, DeKruyff RH. The regulation of allergy and asthma. Immunol Rev 2006:212:238-55.

5. Lambrecht BN, Hammad $\mathrm{H}$. Biology of lung dendritic cells at the origin of asthma. Immunity 2009;31:412-24.

6. Holt PG. Pulmonary dendritic cells in local immunity to inert and pathogenic antigens in the respiratory tract. Proc Am Thorac Soc 2005:2:116-20.

7. Hao X, Kim TS, Braciale TJ. Differential response of respiratory dendritic cell subsets to influenza virus infection. J Virol 2008:82:4908-19.

8. Lambrecht BN, De VM, Coyle AJ, et al. Myeloid dendritic cells induce Th2 responses to inhaled antigen, leading to eosinophilic airway inflammation. J Clin Invest 2000;106:551-9.

9. Liu YJ. IPC: professional type 1 interferon-producing cells and plasmacytoid dendritic cell precursors. Annu Rev Immunol 2005:23:275-306.

10. Gilliet M, Liu YJ. Human plasmacytoid-derived dendritic cells and the induction of T-regulatory cells. Hum Immunol 2002;63:1149-55.

11. Kool M, van NM, Willart MA, et al. An anti-inflammatory role for plasmacytoid dendritic cells in allergic airway inflammation. J Immunol 2009;183:1074-82

12. de Heer HJ, Hammad H, Soullie T, et al. Essential role of lung plasmacytoid dendritic cells in preventing asthmatic reactions to harmless inhaled antigen. J Exp Med 2004;200:89-98.

13. Upham JW, Zhang G, Rate A, et al. Plasmacytoid dendritic cells during infancy are inversely associated with childhood respiratory tract infections and wheezing. J Allergy Clin Immunol 2009;124:707-13.

14. Hiller AS, Tschernig T, Kleemann WJ, et al. Bronchus-associated lymphoid tissue (BALT) and larynx-associated lymphoid tissue (LALT) are found at different frequencies in children, adolescents and adults. Scand J Immunol 1998;47:159-62.

15. Tschernig T, Pabst R. Bronchus-associated lymphoid tissue (BALT) is not present in the normal adult lung but in different diseases. Pathobiology 2000;68:1-8.

16. Bienenstock J, Johnston N, Perey DY. Bronchial lymphoid tissue. I. Morphologic characteristics. Lab Invest 1973;28:686-92.

17. Kocks JR, Davalos-Misslitz AC, Hintzen G, et al. Regulatory T cells interfere with the development of bronchus-associated lymphoid tissue. J Exp Med 2007;204:723-34.

18. Moyron-Quiroz JE, Rangel-Moreno J, Kusser K, et al. Role of inducible bronchus associated lymphoid tissue (BALT) in respiratory immunity. Nat Med 2004; 10:927-34

19. Gould SJ, Isaacson PG. Bronchus-associated lymphoid tissue (BALT) in human fetal and infant lung. J Pathol 1993:169:229-34.

20. Heier I, Malmstrom K, Pelkonen AS, et al. Bronchial response pattern of antigen presenting cells and regulatory T cells in children less than 2 years of age. Thorax 2008;63:703-9.

21. Farkas L, Beiske K, Lund-Johansen F, et al. Plasmacytoid dendritic cells (natural interferon-alpha/beta-producing cells) accumulate in cutaneous lupus erythematosus lesions. Am J Pathol 2001;159:237-43.

22. Tschernig T, De Vries VC, Debertin AS, et al. Density of dendritic cells in the human tracheal mucosa is age dependent and site specific. Thorax 2006;61:986-91.

23. Chieppa M, Rescigno M, Huang AYC, et al. Dynamic imaging of dendritic cell extension into the small bowel lumen in response to epithelial cell TLR engagement. $J$ Exp Med 2006;203:2841-52.

24. Pabst R, Tschernig T. Bronchus-associated lymphoid tissue: an entry site for antigens for successful mucosal vaccinations? Am J Respir Cell Mol Biol 2010;43:137-41

25. Tschernig T, Kleemann WJ, Pabst R. Bronchus-associated lymphoid tissue (BALT) in the lungs of children who had died from sudden infant death syndrome and othe causes. Thorax 1995; 50:658-60.

26. Halle S, Dujardin HC, Bakocevic N, et al. Induced bronchus-associated lymphoid tissue serves as a general priming site for T cells and is maintained by dendritic cells. $J$ Exp Med 2009;206:2593-601. 